\title{
Une nouvelle approche des calculs d'interaction sol-structure
}

\section{GILBERT}

Soletanche entreprise 6, rue de Watford, 92000 Nanterre
Cet article fait la synthèse de la méthode désormais traditionnelle de calculs aux modules de réaction et montre ses insuffisances. Afin de pallier les problèmes ainsi mis à jour, une nouvelle méthode de calculs d'interaction sol-structure est proposée. Ce modèle, dans lequel le sol est modélisé comme un ensemble de ressorts dépendants, est tout aussi simple d'utilisation que celui aux modules de réaction et permet de définir de façon indépendante du chargement les caractérístiques intrinsèques du sol et de la structure. Il permet donc de s'affranchir de la définition d'un module indéfini qui prête toujours à discussion et de se ramener aux propriétés du matériau seul, c'est-à-dire module d'Young et module de cisaillement. Le comportement non linéaire du sol défini par des paliers de plasticité au niveau de la contrainte de cisaillement est aussi intégré au modèle développé, La génération de contraintes de cisaillement permet de mettre en évidence les effets voûtes dans le terrain et ainsi de concentrer les efforts sur les points durs. Basé sur ces principes, un programme de calcul tridimensionnel a été développé afin de traiter les problèmes d'amélioration de terrain par inclusions (rigides ou non rigides) en prenant en compte la couche de répartition éventuelle ainsi que le dallage. Les équations utiles au développement du modèle sont données et des exemples sont traités. En particulier, la notion de module de Westergaard ainsi que la notion d'effet voûte dans les sols sont analysées.

\section{A new approach for soil-structure interaction}

This article summarizes the traditional method of soil-structure interaction based on the modulus of subgrade reaction and shows its weakness. In order to avoid these weakness, a new soil-structure interaction model is proposed. This model considers the soil as a set of connected springs which enables interaction between springs. Its use is as simple as the traditional model but allows to define the soil properties independently from the structural properties and the loading conditions. Thus the definition of the modulus of subgrade reaction is unnecessary as each component is defined by its own modulii (Young's modulus and shear modulus). The non-linear soil behavior for the shear stress versus distortion is also incorporated in the model. This feature allows to pinpoint the arching effect in the ground and shows how the stresses concentrate on stiff materials. Based on these principles, a three dimensional program has been developed in order to solve the difficult problem of soil improvement by inclusions (stiff or soft). Also the possibility to take into account a flexible mat and/or a subgrade layer has been implemented. Equations used in the model are developed and a parametric study of the necessary data used in the program is presented. In particular, the Westergaard modulus notion and the arching effect are analyzed. 


\section{Introduction}

Les calculs actuels de fondations et de soutènements font appel au concept désormais classique de coefficient de réaction. Cette modélisation de l'interaction sol-structure est devenue populaire, car elle permet une modélisation simple du problème à l'aide de logiciels performants et bon marché.

Ce type de calculs appliqués aux soutènements et aux fondations (dallage, pieux, etc.) permet de calculer les déplacements de la structure ainsi que les sollicitations qui s'y appliquent. L'inconvénient majeur de cette méthode est la définition du coefficient de réaction qui dépend d'un grand nombre de paramètres tels la rigidité de la structure et ses dimensions, le chargement, les terrains, etc. et qui, de ce fait, est difficile à définir de manière non équivoque.

Le concept d'interaction sol-structure développé dans cet article part de la nécessité pour le concepteur d'ouvrage d'utiliser un modèle simple et performant tout en ne négligeant pas les concepts de base du géotechnicien qui considère le sol comme un matériau plus complexe qu'un ressort.

\section{2}

\section{La méthode au coefficient de réaction et ses limitations}

Le coefficient de réaction est une notion développée par Westergaard (1938). Il relie de façon conceptuelle la pression, p, appliquée sur le sol à la déflexion, $\delta$, selon l'égalité suivante:

$$
\mathrm{p}=\mathrm{K} \delta
$$

dans laquelle $\mathrm{K}$ est donné en $\mathrm{kPa} / \mathrm{m}$ ou $\mathrm{kN} / \mathrm{m}^{3}$ dans le système SI. De manière complémentaire, le module de réaction, Es, est défini comme le produit du coefficient de réaction, K, par la largeur d'application de la pression, $B$, selon la relation:

$$
\mathrm{Es}=\mathrm{kB}
$$

Le module de réaction est donné en $\mathrm{kPa}$.

Le coefficient de réaction est alors déterminé de façon simple à partir d'un essai de plaque comme montré de façon schématique sur la figure 1 où une courbe de tassement en fonction de la pression appliquée est représentée. La valeur du coefficient de réaction est alors reliée au choix que l'on fait en prenant comme définition du coefficient de réaction soit un coefficient sécant, soit un coefficient tangent, soit encore le coefficient initial comme représenté sur la figure 1. Les valeurs de pression et de tassement obtenues à partir de l'essai de plaque sont des valeurs moyennes; la pression est obtenue comme le rapport de l'effort total appliqué à la surface de la plaque, et le tassement est le tassement moyen mesuré.

A titre d'exemple et afin de faire apparaittre certains paramètres dont dépend le coefficient de réaction, celui-ci est calculé dans le cas d'une plaque circulaire rigide chargée uniformément. La formule de Boussinesq (1885) exprimant le tassement, $\delta$, en fonction de la pression, $\mathrm{p}$, appliquée est la suivante:

$$
\delta=\pi p R\left(1-v^{2}\right) /(2 E)
$$

où $R$ est le rayon de la plaque circulaire, $v$ le coefficient de Poisson et E le module d'Young du matériau élastique.

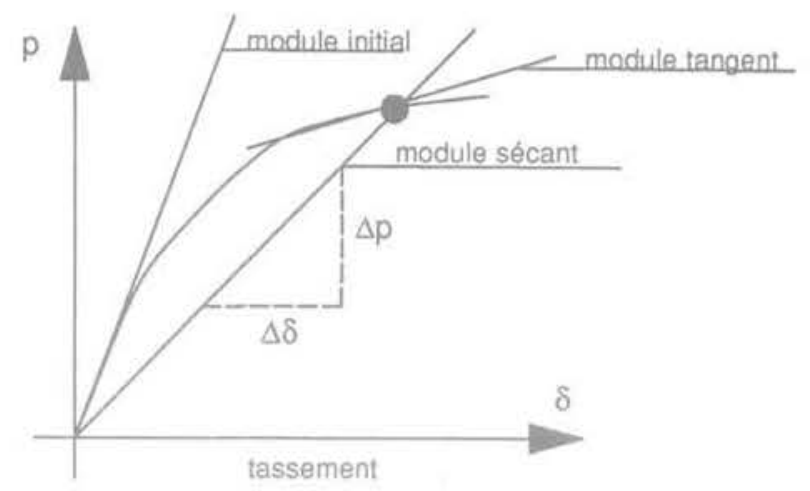

Fig. 1 Définition du coefficient de réaction. Definition of the coefficient of subgrade reaction.

Le tassement devant être le même à partir des équations (1) et (3), le coefficient de réaction est alors égal à :

$$
K=2 E /\left(\pi R\left(1-v^{2}\right)\right)
$$

L'équation (4) montre que dans le cas d'une plaque infiniment rigide, le coefficient de réaction dépend des propriétés élastiques du matériau sol ainsi que des dimensions de la plaque circulaire.

Dans le cas d'une plaque circulaire infiniment souple et chargée uniformément, le coefficient de réaction peut être défini de façon similaire. Le tassement, $\delta_{c}$ au centre de la plaque est donné par:

$$
\delta_{c}=2 \mathrm{p} R\left(1-v^{2}\right) / \mathrm{E}
$$

et le tassement au bord de la plaque, $\delta \mathrm{b}$, est donné par:

$$
\delta_{\mathrm{b}}=1 / 2 \delta_{\mathrm{c}}
$$

Les modules de réaction au centre, $\mathrm{Kc}$, et au bord, $\mathrm{Kb}$, de la plaque infiniment souple sont donnés respectivement par:

$$
K c=E /\left(2 R\left(1-v^{2}\right)\right)
$$

et:

$$
\mathrm{Kb}=1 / 2 \mathrm{Kc}
$$

La comparaison des équations (4), (7) et (8) fait apparaître que le coefficient de réaction dépend aussi de la rigidité de la plaque. Ce phénomène a déjà été discuté et pris en compte par Westergaard dans la définition d'un «rayon de rigidité » et qui correspond pour les poutres à la longueur élastique. Ce rayon, $r_{0}$ s'écrit:

$$
r_{0}=E y h^{3} / 12 /\left(1-v^{2}\right) / K
$$

où $E_{y}$ est le module d'Young du matériau constituant la plaque, $v$ son coefficient de Poisson, h son épaisseur et K le coefficient de réaction du sol sous-jacent.

11 faut noter que dans toutes les formulations le coefficient de réaction est inversement proportionnel au rayon (ou rayon de rigidité). Plus une plaque est rigide, plus son rayon de rigidité augmente et plus le coefficient de réaction diminue. Ceci avait déjà été signalé par Terzaghi (1958) dont la démonstration physique était présentée par l'intermédiaire du concept du bulbe des isocontraintes.

La détermination du coefficient de réaction étant complexe et dépendant de multiples paramètres, la pratique consiste à se fixer des règles empiriques de détermination du coefficient de réaction qui sont basées sur l'expérience. Dans le cas de fondations superficielles, de fondations profondes et de soutènements, le coefficient de réaction est principalement 
déterminé en France à partir des modules pressiométriques. Différentes formulations courantes sont actuellement utilisées en fonction du type d'ouvrage (pieu ou soutènement) et du type de sollicitations (mise en tension de tirants, excavation, etc.) ainsi que de la vitesse d'application. A titre d'exemple nous citerons les plus courantes.

La formule de base pour la détermination du coefficient de réaction consiste à appliquer directement le résultat de l'essai pressiométrique. Cette formule donne le coefficient de réaction d'une section en arc de cercle de rayon $\mathrm{R}$ :

$$
\mathrm{K}=\mathrm{E}_{\mathrm{m}} /[(1+\mathrm{v}) \mathrm{R}]
$$

où $\mathrm{E}_{m}$ est le module pressiométrique. Cette formule diffère de la formule donnée par l'application des concepts de l'élasticité car le sol autour de l'excavation se plastifie. Dans un milieu purement élastique, le coefficient de réaction est donné par:

$$
K=E /[(1+v) R]
$$

où $\mathrm{E}$ est et le module d'Young du matériau élastique. Pour ce qui est des fondations superficielles de longueur, L, et de largeur, B, le coefficient de réaction, K, est donné à partir des formules pressiométriques par:

$$
\mathrm{K}=9 \mathrm{E}_{\mathrm{m}} /\left(2 \mathrm{~B}_{0}\left(\lambda_{\mathrm{d}} \mathrm{B} / \mathrm{B}_{0}\right)^{\alpha}+\alpha \lambda_{\mathrm{c}} \mathrm{B}\right)
$$

où $B_{0}$ est la largeur de référence, $\lambda_{f}$ et $\lambda_{f}$ les coefficients de forme, fonction du rapport L/B.

Pour ce qui est des pieux dont le diamètre, $B$, est supérieur à $0,60 \mathrm{~m}$, le module de réaction sous effort horizontal est donné par les formules suivantes :

$$
\mathrm{Es}=18 \mathrm{E}_{\mathrm{m}} /\left[4\left(2,65\left(\mathrm{~B} / \mathrm{B}_{0}\right) \propto \mathrm{B}_{0} / \mathrm{B}+3 \alpha\right]\right.
$$

où $B_{0}$ est le diamètre de référence égal à $0,60 \mathrm{~m}, \alpha$ le coefficient rhéologique et $\mathrm{E}_{\mathrm{m}}$ le module pressiométrique.

Lorsque le diamètre du pieu est inférieur ou égal à $0,60 \mathrm{~m}$ alors le module de réaction est donné par :

$$
\mathrm{Es}=18 \mathrm{E}_{\mathrm{m}} /\left[4(2,65)^{\alpha}+3 \alpha\right]
$$

Pour la détermination du coefficient de réaction pour les calculs de soutènement, un certain nombre de formules sont actuellement utilisées dont les principales sont:

- Menard-Bourdon (1965):

$$
\mathrm{K}=\mathrm{E}_{\mathrm{m}} /\left(\alpha \mathrm{a} / 2+0,133(9 \mathrm{a})^{\alpha}\right)
$$

où a est un paramètre relié à la longueur mise en butée. - Balay (1980), mise en tension des tirants:

$$
\mathrm{K}=\operatorname{rs} 4 \mathrm{E}_{\mathrm{m}} / \mathrm{L}_{0}
$$

avec:

$$
\mathrm{L}_{0}=\left(\mathrm{ELI}_{\mathrm{m}}\right)^{1 / 3}
$$

et $r$ et s sont des coefficients tenant compte de la méthode de mise en place ainsi que de la géométrie.

La méthode pénétrométrique peut aussi être utilisée afin de fournir des corrélations entre le module de réaction horizontal et la résistance en pointe, $\mathrm{q}_{c}$. A titre indicatif une formule empirique couramment utilisée est la suivante:

$$
\text { Es }=4,5 q_{c}
$$

Un certain nombre d'auteurs tels que Terzaghi (1955), Rowe (1957), Gigan (1989) ont donné des relations empiriques afin d'évaluer le coefficient de réaction en fonction de paramètres tels que la cohésion non drainée, la profondeur, la fiche, etc. Une formulation couramment admise dans la pratique pour l'évaluation des coefficients de réaction horizontaux destinés aux calculs des soutènements est donnée par Chadeisson
(1975) sous forme d'abaques reliant le coefficient de réaction à l'angle de frottement et à la cohésion du terrain.

D'une manière générale, Schmitt (1984) a mis en évidence expérimentalement la sous-évaluation systématique des coefficients de réaction déterminés par les méthodes traditionnelles lorsque les déformations sont faibles. Ce constat est identique à celui effectué sur le module d'Young.

L'utilisation de l'équation (1) montre que la déformée du sol seul, chargé avec une pression $p(x)$ où $x$ est l'abscisse, est homothétique au chargement. De façon explicite, la déformée du sol, s(x), s'écrit:

$$
\mathrm{s}(\mathrm{x})=\mathrm{p}(\mathrm{x}) / \mathrm{K}
$$

La figure 2 montre la déformée du sol représentée par un coefficient de réaction sous une bande uniformément chargée de rigidité nulle et la compare au tassement d'un matériau élastique soumis à ce même chargement.

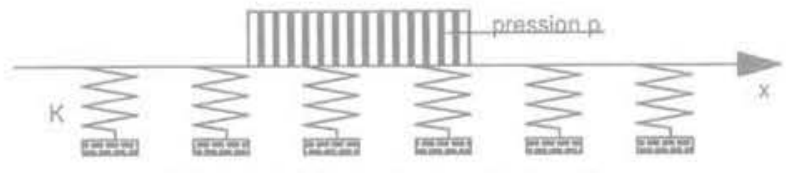

(a) Modélisation du sol et du chargement

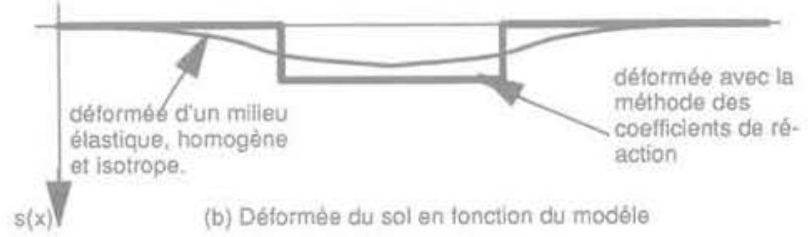

FiG.9 Exemple de chargement et déformée selon le modèle pris en compte. Example of settlement curves.

Il apparaît dans la figure 2 que le sol modélisé à partir des coefficients de réaction en dehors de la zone chargée ne subit aucune déformation contrairement à la modélisation du sol comme milieu homogène, élastique et isotrope. De plus, la déformée obtenue à partir de la modélisation aux modules de réaction est discontinue et fortement éloignée de la déformée réelle, quelle que soit la valeur du module retenu.

Cet ensemble de formules empiriques simplifie énormément le travail du projeteur au prix d'une modélisation trop ( simpliste » qui fait oublier l'existence du matériau sol au comportement plus complexe. Afin de prendre en compte le sol, un modèle d'interaction simple est développé.

\section{3 \\ Le modèle d'interaction sol-structure: GATEM}

Le point négatif de la méthode aux modules de réaction est l'absence de couplage des ressorts adjacents. La modélisation que nous proposons consiste à connecter les ressorts adjacents par des éléments de type «contact » et à établir une loi d'interaction.

Afin d'établir les équations nécessaires, considérons deux ressorts comme indiqué sur la figure 3 . 
Noeud 1

Noeud 2
K1

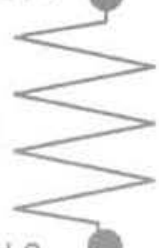

Interaction entre deux ressorts adjacents.

Adjacent springs interaction.

Ces ressorts représentent des colonnes de sol comme indiqué en figure 4. La matrice de rigidité élémentaire de chaque ressort est la suivante.

$$
K_{\mathrm{C}}=\frac{\mathrm{ES}}{\mathrm{L}}\left[\begin{array}{cc}
1 & -1 \\
-1 & 1
\end{array}\right]
$$

où le facteur ES/L représente la rigidité $\mathrm{K}$ du ressort. E est le module d'Young du sol, S la section de la colonne de sol considérée et $\mathrm{L}$ sa longueur.

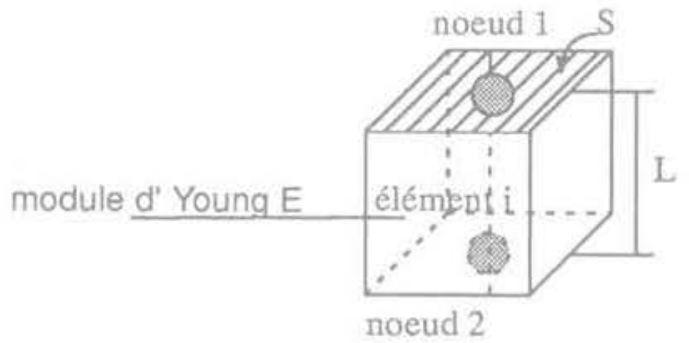

MG.4 Représentation de l'élément de sol représenté par le ressort de rigidité $\mathrm{K}$.

Soil column modeled as a spring.

En plus du terme de rigidité axiale, il existe une interaction entre les éléments voisins $i$ et j comme montré sur la figure 5. Afin d'établir les équations nécessaires, nous supposerons un comportement linéaire élastique entre ces deux éléments. Soit p la largeur de contact entre ces deux éléments et $\mathrm{L}$ la hauteur de contact, le déplacement relatif entre les nœuds du sommet, 1 et 3, ainsi qu'entre les nouds du bas est défini par:

$$
\begin{aligned}
& -\Delta u_{1}=u_{3}-u_{4} \\
& -\Delta u_{2}=u_{4}-u_{2}
\end{aligned}
$$

où u est le déplacement du nœud i. En appelant $\mathrm{k}$ la rigidité reliant la contrainte de cisaillement, $\tau$, au déplacement relatif, $\Delta \mathrm{u}$,

$$
\tau=k \Delta u
$$

la matrice de rigidité élémentaire pour le comportement élastoplastique de l'élément dans le plan XOZ ou YOZ s'écrit:

$$
K_{e}=C\left[\begin{array}{ccrr}
2 & 1 & -2 & -1 \\
1 & 2 & -1 & -2 \\
-2 & -1 & 2 & 1 \\
-1 & -2 & 1 & 2
\end{array}\right]
$$

avec:

$$
C=\frac{p L k}{6}
$$

Dans le cas d'un comportement élasto-plastique des contacts, la matrice de rigidité a une forme similaire à celle donnée précédemment, mais la valeur k est choisie comme la valeur moyenne trouvée aux nœuds inférieurs et supérieurs. Dans ce cas, la matrice est évaluée à partir d'un module sécant, et cette formulation permet de garder une matrice de forme symétrique qui simplifie le traitement des équations ainsi que le stockage.

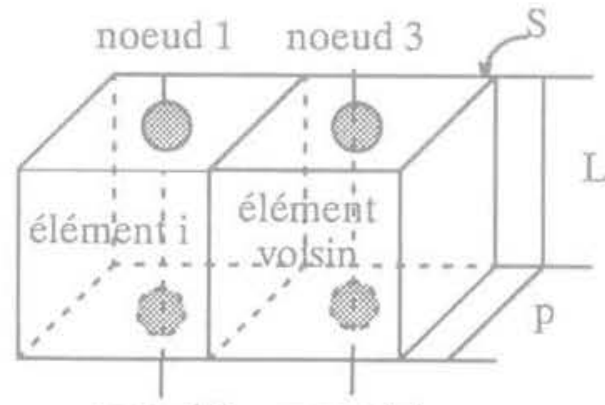

noeud 2 noeud 4

FGG.3 Représentation de deux éléments adjacents et numérotation. Adjacent soil elements.

\section{4}

\section{Applications de ce modèle à quelques cas simples}

Les concepts de ce modèle ont été mis en ceuvre dans le programme GATEM (Gilbert et al., 1993) qui est un logiciel traitant de l'amélioration des sols mous par inclusions. Il est destiné à résoudre les problèmes sujvants qui sont montrés sur la figure 6 .

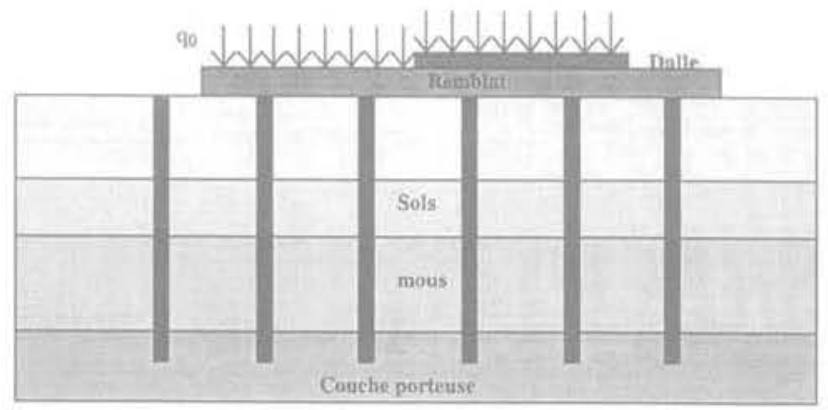

FiG.6 Champs d'application du programme GATEM.

Problems dealt with by GATEM.

En particulier, l'utilisation d'inclusions plus ou moins rigides comme des pieux, des colonnes de jet grouting, des colonnes ballastées, des puits ballastés, 
etc. peut être modélisée pour voir leur rôle quant à l'amélioration de terrains mous.

Deux exemples d'application seront traités ici correspondant aux problèmes bien connus de la détermination du module de Westergaard, ainsi qu'à la représentation de l'effet de voûte qui se développe dans le sol lors de la présence de "points durs ».

Le premier exemple correspond à un essai de plaque dans lequel les dimensions de la plaque sont des paramètres variables. Le cas étudié correspond à une surface de sol de $25 \mathrm{~m} \times 25 \mathrm{~m}$ composé d'une couche de $10,0 \mathrm{~m}$ d'épaisseur surmontée par un remblai de $0,50 \mathrm{~m}$ d'épaisseur.

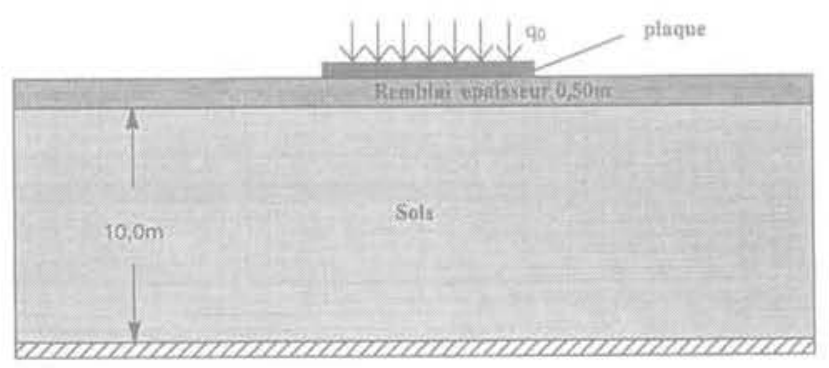

FHG.7 Géométrie de l'exemple 1.

Geometry for example 1.

Les caractéristiques mécaniques prises en compte pour les deux couches sont données dans le tableau I.

TABLEAUI Caractéristiques mécaniques prises en compte pour l'exemple 1 . Soil properties for example 1.

\begin{tabular}{|c|c|c|c|c|}
\hline Terrain & $\begin{array}{c}\text { Epaisseur } \\
\text { (m) }\end{array}$ & $\begin{array}{c}\text { Moxule } \\
\text { dYoung (MPa) }\end{array}$ & $\tau_{\text {( }}^{\tau_{\text {mal }}}$ & (min) \\
\hline Remblai & 0,50 & 70 & 100 & 0,01 \\
\hline Argile & 10,0 & 10 & 100 & 0,01 \\
\hline
\end{tabular}

Le comportement à l'interface des colonnes de sols est considéré comme élastoplastique. Plus explicitement, la contrainte de cisaillement varie comme indiqué sur la figure 8 en fonction du déplacement relatif.

Des plaques carrées de $1,0 \mathrm{~m}, 3,0 \mathrm{~m}, 5,0 \mathrm{~m}, 15,0 \mathrm{~m}$ et $25,0 \mathrm{~m}$ ont été chargées sous une contrainte de $70 \mathrm{kPa}$ et

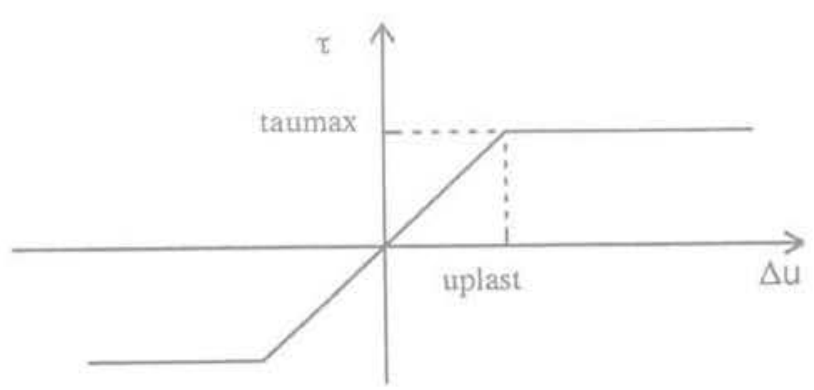

FG.8 Relation entre les contraintes de cisaillement et le déplacement relatif à l'interface sol/sol.

Stress-strain law for the contact element.

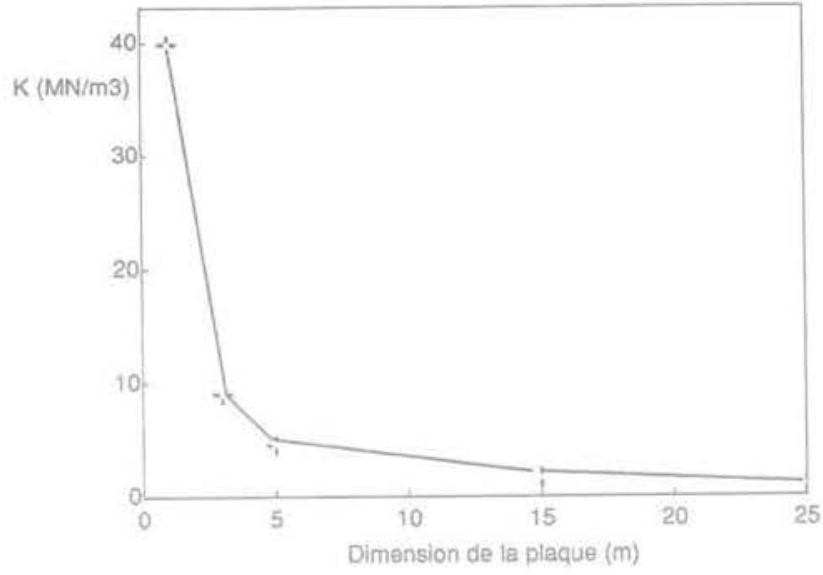

RG.9 Evolution du coefficient de réaction en fonction des dimensions de la zone chargée.

Coefficient of subgrade reaction as a function of plate width.

le coefficient de réaction en fonction des dimensions de la plaque est donné sur la figure 9.

Comme les formules basées sur l'élasticité le laissent présager, le coefficient de réaction diminue lorsque la surface chargée augmente. De plus, quand la dimension de la zone chargée augmente, le coefficient de réaction tend vers la valeur suivante:

$$
\mathrm{K}=\mathrm{E}_{y} / \mathrm{H}
$$

où $E_{y}$ correspond au module de l'ensemble remblai plus sol (moyenne harmonique) et $\mathrm{H}$ correspondant à l'épaisseur totale de la couche. Dans le cas de l'exemple 1. le module d'Young moyen est égal à $10,42 \mathrm{MPa}$, et le coefficient de réaction est alors égal à $0,99 \mathrm{MN} / \mathrm{m}^{3}$.

Le deuxième exemple consiste à déterminer le report d'effort sur des inclusions rigides de type pieux lorsque ceux-ci sont utilisés pour renforcer un terrain compressible. La géométrie du problème est donnée en figure 10, et les caractéristiques mécaniques retenues pour cet exemple sont données dans le tableau II.

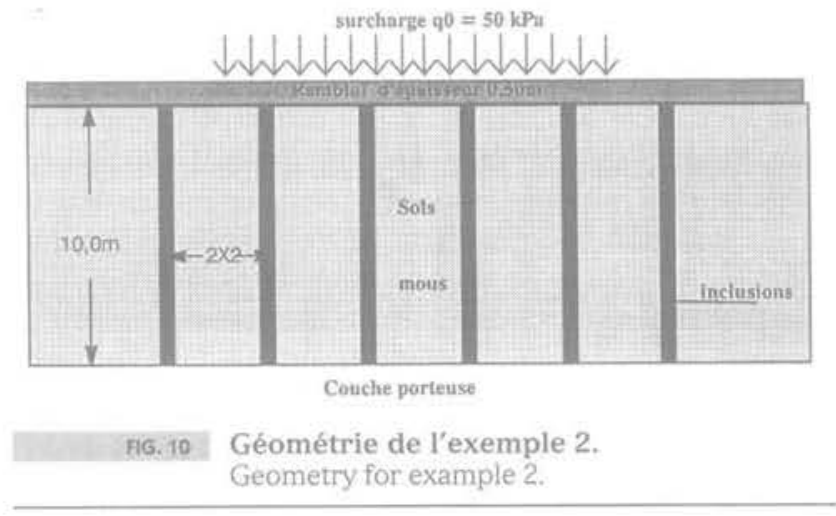

Le sol est chargé avec une surcharge uniforme de $50 \mathrm{kPa}$. Le sol compressible est renforcé par des inclusions en béton de diamètre $0,27 \mathrm{~m}$ réparties selon une maille carrée de $2,0 \mathrm{~m}$ qui sont fíchées dans le substratum.

L'utilisation d'inclusions va permettre au sol de se décharger par mise en jeu du frottement négatif sur les 
TaBLEAU II Caractéristiques mécaniques prises en compte pour l'exemple 2.

Soil characteristics for example 2.

\begin{tabular}{|c|c|c|c|c|c|c|}
\hline \multirow[t]{2}{*}{ Temran } & \multirow[t]{2}{*}{$\begin{array}{c}\text { Épaisseur: } \\
\text { (m) }\end{array}$} & \multirow[t]{2}{*}{$\begin{array}{c}\text { Module } \\
\text { d'Young (MPal }\end{array}$} & \multicolumn{2}{|c|}{$\begin{array}{c}\text { Interaction } \\
\text { solsol }\end{array}$} & \multicolumn{2}{|c|}{$\begin{array}{c}\text { Interaction } \\
\text { solpiey }\end{array}$} \\
\hline & & & $\begin{array}{c}\tau \\
(\mathrm{kPa})\end{array}$ & $\begin{array}{l}\mathrm{U}_{\text {phos }} \\
(\mathrm{m})\end{array}$ & $\tau_{(\mathrm{kpax})}^{\tau_{\max }}$ & $\begin{array}{l}U_{\text {plas }} \\
\mathrm{m} \text { ) }\end{array}$ \\
\hline Remblai & 0,50 & 70 & 100 & 0,01 & 100 & 0,01 \\
\hline Argile & 10,0 & 6 & 50 & 0,01 & 30 & 0,01 \\
\hline
\end{tabular}

pieux. Les pieux vont donc se charger jusqu'à une certaine profondeur (point de frottement négatif nul), et le sol va se décharger. La conséquence de ce transfert de charge est la diminution du tassement de la couche compressible. Dans l'exemple traité, le point de frottement négatif nul se situe à la base de la couche compressible comme nous le verrons dans l'analyse des résultats.

Dans cet exemple, on suppose que l'interaction sol/inclusion est identique quel que soit le signe des contraintes de cisaillement (frottement positif ou négatif). Le module d'Young des inclusions est pris égal à $10000 \mathrm{MPa}$.

Les résultats obtenus sont présentés en figures 11 et 12. La figure 11 représente le tassement en surface sous $50 \mathrm{kPa}$ et le compare au tassement sans amélioration. Les tassements sont réduits dans un rapport de l'ordre de 3,3 , ce qui correspond à une valeur usuelle dans ce type de traitement.

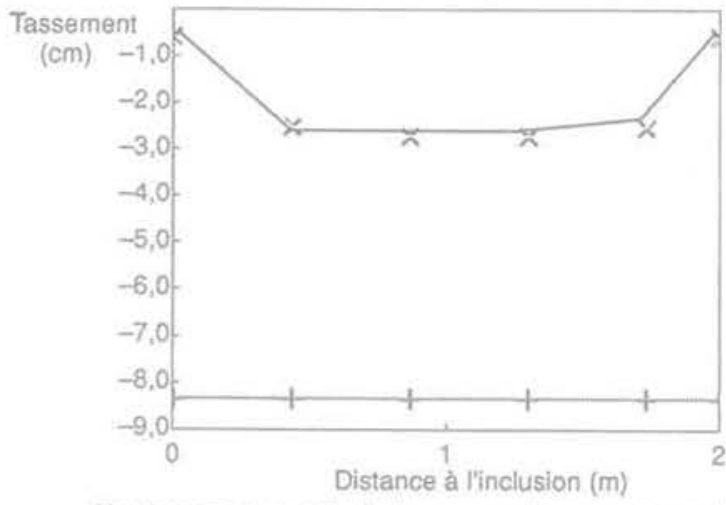

$X$ : tassement avec inclusions + ; tassement sans inclusion

Tassement en surface en fonction de la distance à l'inclusion.

Surface settlement.

De même, la variation de contrainte dans l'inclusion en fonction de la profondeur est donnée en figure 12 où il apparaît que l'inclusion se charge en profondeur par effet du frottement négatif.

Le phénomène de déchargement du sol est représenté sur la figure 13 pour différentes distances à l'inclusion. Partant d'une contrainte de 0,05 MPa en surface, les contraintes verticales diminuent quand la profondeur augmente et sont plus importantes lorsque la distance à l'inclusion est plus grande. Ce phénomène est compatible avec le fonctionnement proposé par Combarieu.

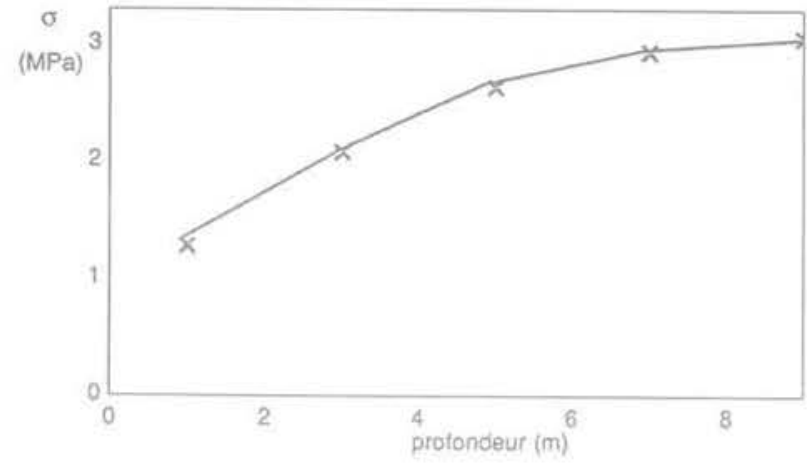

7G. 12 Contrainte dans les inclusions en fonction de la profondeur.

Compressive stress in the inclusion.

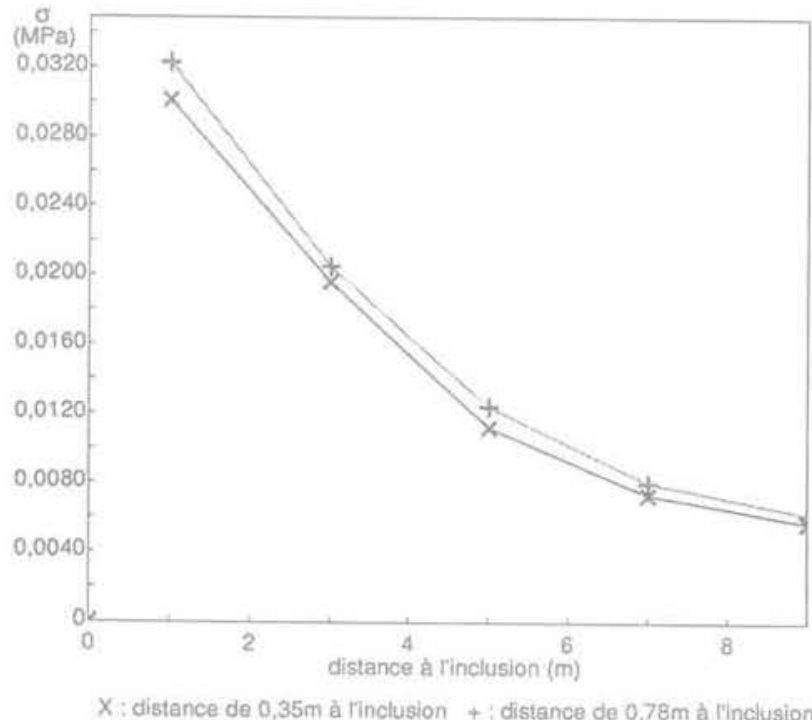

Fis.13 Contrainte dans le sol en fonction de la profondeur pour différentes distances à l'inclusion.

Vertical stress in the soil as a function of the distance to the inclusion.

\section{Conclusions}

Le modèle de calcul d'interaction sol-structure présenté dans cet article permet par l'intermédiaire d'un artifice de modélisation de représenter le comportement du sol plus exactement que par la méthode traditionnelle du module de réaction. Les changements de notions qu'il envisage sont relativement faibles et pourront certainement s'intégrer dans la continuité du travail des bureaux d'études sans révolutionner leurs approches. Cette méthode plus réaliste est à mi-chemin entre la méthode trop simpliste du module de réaction et la méthode plus complexe des éléments finis. De plus, cette notion, appliquée au traitement du délicat problème d'amélioration de terrain, permet de quantifier les améliorations provoquées. Ce modèle pourra s'appliquer aux calculs des soutènements et permettra, sans aucun doute, de tenir compte des phénomènes d'effet de voûte actuellement totalement occultés. 
Balay J. - Recommandations pour le choix des paramètres de calcul des écrans de soutènement par la méthode aux modules de réaction, LCPC. FAEF 1. $07.12,1984$

Chadeisson - Note interne Solétanche pour la définition des coefficients de réaction, 1975.

Gigan J.-P. - Expérimentation d'un ridear de palplanches ancré par tirants actifs, Bulletin de liaison des laboratoires des Ponts et Chaussées, n 129, 1984.

Gilbert C., Anfrie E. - Notice technique du programme GATEM et Notice d'utilisa- tion du programme GATEM, Documents internes Solétanche, 1993.

Marche R. - Sollicitation en flexion des pieux par les couches qu'il traverse, Thèse de doctorat, Ecole polytechnique de Lausanne, 1974.

Menard L., Bourdon C. - Calcul des rideaux de soutènement. Méthode nouvelle prenant en compte les conditions réelles d'encastrement, Sols-Soils, $n^{\circ} 12$, 1965.

Rowe P.W. - A theoritical and experimental analysis of sheetpile wails, Proceedings of ICE, 1957.
Schmitt P. - Étude expérimentale de la sollicitation exercée par le sol sur les ouvrages de soutènements souples, Revue Française de Géotechnique, $n^{\circ} 28$, 1984.

Terzaghi K - Evaluation of coefficients of subgrade reaction, Geotechnique, 1955. Westergaard H.M. $-\alpha$ A problem of Elasticity Suggested by... s) in Contributions to the Mechanics of Solids, Stephen Timoshenko 60th Anniversary Volume, The Macmilan Co, NY, 1938. 